\title{
TELEOLOGÍA Y NATURALEZA EN ARISTÓTELES
}

\author{
Alberto Ross ${ }^{1}$ \\ Facultad de Filosofía, Universidad Panamericana (México)
}

Recibido: 08.10.2018 - Aceptado: 16.11.2018

\begin{abstract}
RESUMEN
El propósito de este trabajo es presentar una reconstrucción de cómo se articulan las nociones de finalidad y naturaleza en la física de Aristóteles, así como de la relación que guardan estos principios con la substancia eterna e inmóvil que introduce el filósofo griego en Metaph. XII. Como es bien sabido, hay un espectro amplio de lecturas acerca de la postura aristotélica sobre el particular, las cuales responden a distintas valoraciones de los argumentos que aparecen en los pasajes que el filósofo griego dedica al estudio de estos temas. La lectura que se defenderá en el presente texto podría describirse como minimalista.
\end{abstract}

Palabras clave: Aristóteles; física; metafísica; naturaleza; causa; finalidad.

\begin{abstract}
The aim of this paper is to offer an account of the relationship between teleology and nature in Aristotle's physics. In order to achieve this purpose, the article comprises two parts. Firstly, the reconstruction of the Aristotelian arguments that justify the thesis according to which "nature belongs to the kind of causes that act for the sake of something." Secondly, the explanation of the role played by the eternal and immobile substance of Metaph. XII in this account. There are different interpretations about this point, but this article defends a minimalistic position about the connection between the prime unmoved mover and the natural order.
\end{abstract}

Keywords: Aristotle; physics; metaphysics; nature; cause; teleology.

\footnotetext{
1 jaross@up.edu.mx
} 


\section{INTRODUCCIÓN}

El propósito de este trabajo es presentar una reconstrucción de cómo se articulan las nociones de finalidad y naturaleza en Aristóteles, así como de la relación que guardan estos principios con la substancia eterna e inmóvil que introduce el filósofo griego en Met. XII. Como es bien sabido, a propósito de este tema hay un amplio espectro de lecturas maximalistas y minimalistas de la postura aristotélica sobre el particular, las cuales responden a distintas valoraciones de los argumentos que aparecen en los pasajes que el filósofo griego dedica al estudio de estos temas. La lectura que defenderé en el presente texto podría catalogarse como minimalista.

En aras de atender a lo que nos hemos propuesto, primero explicaré cómo surge la cuestión que nos ocupa a partir de la necesidad de dar cuenta de la relación entre algunos pasajes polémicos del corpus aristotelicum. Presentaré, por tanto, algunos textos cuya interpretación y articulación han dado lugar a posturas de distinta índole. Una vez hecho esto, pasaré al análisis de algunos textos que son claves para la definición del tema que nos ocupa. Primero, presentaré la respuesta que ofrece Aristóteles en $P h$. II 8 a la pregunta de por qué la naturaleza se encuentra entre las causas que son para algo.2 Después, explicaré cómo hay que entender la relación de "dependencia" que se puede establecer entre una phýsis orientada teleológicamente como la que concibió el filósofo griego y una substancia eterna e inmóvil separada del mundo físico. Mi propuesta al respecto tiende a ser más bien deflacionaria.

\section{LA NATURALEZA Y LA SUBSTANCIA ETERNA E INMÓVIL}

A lo largo de la historia del aristotelismo y su transmisión, podemos encontrar interpretaciones minimalistas y maximalistas de la articulación de la noción de télos con otras nociones claves del aristotelismo como las de phýsis, agathós o theós. Podemos encontrar esta diversidad de lecturas a propósito del tema desde los comentadores antiguos hasta nuestros días, ya sea entre la literatura especializada o entre los autores que apelan al filósofo griego como antecedente de alguna postura específica en el marco de argumentaciones sistemáticas. La reconstrucción de los textos aristotélicos puede dar lugar a distintos tipos de teorías acerca de cómo se articulan las nociones de naturaleza, lo divino y el bien, teniendo a la teleología como uno de sus hilos conductores.

\footnotetext{
${ }^{2}$ Cfr. Ph.198b10-11.
} 
Recordemos que Aristóteles, en Metaph. XII, argumenta a favor de la existencia de una substancia eterna e inmóvil, que se caracteriza por ser inmaterial, pura actividad, mover con un movimiento único y sin ser movido y hacerlo como lo deseable y lo inteligible. ${ }^{3}$ En este orden de ideas, Aristóteles señala que "de tal principio penden el cielo y la naturaleza" 4 , dicho lo anterior. explica en qué consiste la vida de este principio y lo identifica con dios o lo divino. ${ }^{5}$ ¿Cómo entender esta relación de dependencia del cielo y la naturaleza respecto del principio ya descrito? Éste es, quizás, uno de los puntos que ha generado más posibilidades en el espectro de respuestas maximalistas y minimalistas ya mencionado.

A lo largo de la historia de la recepción de la filosofía aristotélica, el pasaje anterior ha sido relacionado con otros textos del corpus como el ya referido $P h$. II. Ahí, el filósofo griego define a la naturaleza en II 1 como "cierto principio y causa del movimiento o del reposo en aquello que es inherente primeramente y por sí, es decir, no por accidente"6 y distingue lo que significa "por naturaleza", "según naturaleza", ${ }^{7}$ así como los sentidos de phýsis. ${ }^{8}$ Todas estas distinciones juegan un papel muy importante en la argumentación de por qué la naturaleza se encuentra entre las causas que son para algo en II $8 .^{9}$

Una postura maximalista trataría de relacionar la doctrina de $P h$. II con el pasaje de Metaph. XII que señala la conexión entre la substancia eterna y la naturaleza. De esta forma, podría atribuírsele a la substancia eterna, i.e. a dios, la causalidad propia y última de la orientación a fines de la naturaleza y, por tanto, de todo lo que es por naturaleza y conforme a la naturaleza. En consecuencia, se le podría atribuir también cierta noción de providencia a dicho principio divino, como lo han hecho algunos autores. Mencionaré algunos ejemplos, pero antes podríamos añadir un énfasis adicional.

A la reconstrucción anterior, podrían sumarse algunos textos de la Ética en aras de abonar a la aproximación de las obras de filosofía teórica y de filosofía práctica. Pensemos, por ejemplo, en el argumento del érgon en EN I 6 y a los pasajes de V 7 donde se habla de lo justo por naturaleza. Dada esta vinculación, a la versión maximalista ya señalada se le podría añadir un matiz adicional: dado que el cielo y la naturaleza dependen de la substancia eterna e inmóvil que se identifica con dios, entonces éste sería la causa última de que la naturaleza se encuentre entre las causas que son para algo y, por tanto, sería el fundamento

\footnotetext{
${ }^{3}$ Cfr. Metaph. 1071b3-27.

${ }^{4}$ Cf. Metaph. 1072b13-14.

${ }^{5}$ Cfr. Metaph. 1072b4ss.

${ }^{6}$ Ph. 192b21-23. Sigo la traducción de Marcelo Boeri en este pasaje y los subsiguientes, cuyos datos bibliográficos se recogen al final de este trabajo.

${ }^{7}$ Cfr. Ph. 192b32-193a2.

${ }^{8}$ Cfr. Ph. 193a9-193b21.

${ }^{9}$ Cfr. Ph. 198b10ss.
} 
último de todo aquello que es por naturaleza y según naturaleza. Por ejemplo, lo justo. Así, tendríamos una versión providencialista y iusnaturalista de Aristóteles en sentido fuerte.

El espectro de la literatura secundaria que suscribe total o parcialmente una reconstrucción cercana a ésta es muy amplio y, por supuesto, con muchos matices. Un ejemplo célebre de este tipo de lectura podemos encontrarlo en Alasdair MacIntyre quien sostiene que la identificación del érgon del hombre como el ejercicio de los poderes racionales se presenta en EN I "como si fuera evidente, y lo es sobre el fondo de la visión aristotélica del universo". ${ }^{10}$ Para MacIntyre, dicho argumento es inteligible en un contexto muy peculiar y que no es asequible, a primera vista, para cualquier persona. En su explicación de este punto, la visión del universo que haría inteligible el argumento, según MacIntyre, es la siguiente: "La naturaleza se compone de tipos de seres bien demarcados y distintos, y cada uno se mueve y es movido desde su potencialidad a ese estado de actividad en que alcanza su meta. En la cima de la escala se encuentra el motor inmóvil, inmutable y pensante, hacia el que se mueven todas las cosas. El hombre, como cualquier otra especie, se mueve hacia una meta, y esa meta puede determinarse mediante la simple consideración de lo que lo diferencia de las demás especies. Dada la visión general, la conclusión parece inatacable; pero sin ella parece muy improbable". ${ }^{11}$ La comprensión cabal del argumento del érgon supondría cierta familiaridad con este cuadro, que incluye tesis desarrolladas por Aristóteles tanto en su Physica como en su Metaphysica. Es importante destacar que no sólo sostiene que la explicación última de que la función propia del hombre sea el buen uso de las capacidades racionales radica en la visión teleológica de Aristóteles, sino que piensa que el argumento no es inteligible al margen de una reconstrucción maximalista de ella. De manera que dicho trasfondo se presenta como un principio tanto en el orden del ser como del conocer. Esto contrasta, de entrada, con otras interpretaciones que sostienen que el argumento es inteligible al margen de las obras y las doctrinas de metafísica, psicología o filosofía de la naturaleza del Estagirita. No puedo entrar en esa discusión ahora, aunque he intentado fijar mi postura en otro lugar a favor de una lectura minimalista de las relaciones entre ética y metafísica en Aristóteles. ${ }^{12}$

Si se me permite añadir un ejemplo adicional de interpretaciones máximalistas, pero de otro corte y un poco más moderada, podemos referirnos a la reconstrucción de la doctrina aristotélica que hizo Alejandro de Afrodisia. En particular, a la que se refiere a la relación entre la substancia eterna e inmóvil y

\footnotetext{
${ }^{10}$ Cfr. MacIntyre 1991, 69.

${ }^{11}$ Cfr. MacIntyre 1991, 69.

${ }^{12}$ Cfr. Ross 2013, 127-153.
} 
la naturaleza. El cuadro explicativo en sede alejandrina es un poco más complicado, incluso que el propuesto por el mismo Aristóteles. Alejandro retoma en el de Fato algunas de las nociones claves de Ph. II (naturaleza, causa, azar y finalidad), pero añade algunas más como la de destino (heimarméne). De acuerdo con el comentarista antiguo, todo lo que sucede conforme a la naturaleza, ocurre también conforme al destino. Sin embargo, el poder causal de ambos no es universal. Alejandro excluye del dominio del destino lo que sucede por accidente, i.e. por azar o por fortuna y lo que procede de la deliberación. ${ }^{13}$ Además, Alejandro añade en Mantissa 25 que tampoco es aceptable decir que las cosas eternas suceden de acuerdo con el destino y lo ilustra con algunos ejemplos: el que un triángulo tenga sus ángulos internos iguales a dos rectos, ${ }^{14} \mathrm{el} \mathrm{movi-}$ miento del sol, el solsticio de verano o el movimiento de los cuerpos celestes ${ }^{15}$ no ocurren de acuerdo con el destino. Éste no es causa de las cosas eternas o de las que suceden siempre de la misma manera, sino que su causalidad se da solamente en el mundo sublunar y es coextensivo con todo aquello que sucede de acuerdo con la naturaleza. Por eso también lo que sucede por azar, fortuna o deliberación queda fuera de su ámbito explicativo, así como lo que es causado por la providencia. Tenemos pues, un cuadro explicativo mucho más complejo que el aristotélico.

La pronoía, para Alejandro, es causa del movimiento del cielo, pero su acción también alcanza al mundo sublunar y no en un sentido accidental (como si se dijera que el ciclo eterno de la generación y corrupción que se da en el mundo sublunar es un efecto no intentado de una actividad principal, a saber, la de mover eternamente al cielo, que sería el objeto directo de su acción). ${ }^{16} \mathrm{Si}$ bien en $P h$. VIII, Aristóteles defiende que que el Primer Motor no puede ser causa directa de lo que sucede en el mundo sublunar, ${ }^{17}$ Alejandro señala que la providencia se extiende justo hasta el mundo sublunar haciendo uso precisamente del movimiento de los cuerpos celestes para mantener el ciclo eterno de generaciones y corrupciones, aunque esta acción no se extiende hasta los individuos. ${ }^{18}$ En el De fato, Alejandro sostiene que la configuración específica de las entidades divinas de acuerdo con su movimiento en relación a las cosas del mundo sublunar es el principio de cada generación. ${ }^{19}$ Esta relación sería propia, no accidental. De manera que la relación dios-naturaleza en Alejandro sería más estrecha que la defendida por la interpretación tradicional de Aristóteles que

\footnotetext{
${ }^{13}$ Cfr. Alejandro de Afrodisia, De Fato, 167-174.

${ }^{14}$ Cfr. Alejandro de Afrodisia, Mantissa 25, 181, 4-14.

${ }^{15}$ Cfr. Alejandro de Afrodisia, Mantissa 25, 181, 14-28

${ }^{16}$ Cfr. Quaest. 2.21, 65.17ss.

${ }^{17}$ Cfr. Ph.260a.3-17.

${ }^{18}$ Cfr. De prov. 33.1ss. y 87.1ss; Quaest. 1.25, 41.8-19.

${ }^{19}$ De fato $169,25-26$.
} 
defiende que el efecto de la acción divina sólo recae, propiamente hablando, en el primer movido.

A partir de los ejemplos anteriores, tenemos dos muestras de botón de distintas formas de explicar cómo se da el reino de los fines en la naturaleza y su conexión con las substancias separadas o, particularmente, con dios o lo divino. En lo que concierne a este trabajo, como ya dijimos, trataré de defender una versión más moderada o minimalista de la interpretación de la postura de Aristóteles sobre estos temas. Para ello, veremos a continuación la reflexión de Aristóteles acerca de por qué la naturaleza se cuenta entre las causas que son para algo y después regresaremos al pasaje de Metaph. XII 7.

\section{NATURALEZA Y TELEOLOGÍA EN PH. II 8}

Aristóteles dice, en $P h$. II 7, que: "puesto que las causas son cuatro será tarea del físico conocerlas todas y haciendo referencia a todas ellas -a la materia, a la forma, al motor y al fin-, podrá responder al porqué de un modo físico”. ${ }^{20} \mathrm{El}$ contexto general de esta reflexión es la determinación del tipo de explicaciones que ofrece la ciencia de la naturaleza. Obviamente, la prescripción de Aristóteles recogida en el pasaje necesita ser aclarada, pues no bastaría decir que las causas o explicaciones son de cuatro tipos para decir que la ciencia de la naturaleza debe remitirse a todas ellas. Si una ciencia como las matemáticas se remitiera a una sola de ellas porque así conviene, v.gr. a la formal, esto no sería un inconveniente. ${ }^{21}$ La pregunta que se debe plantear entonces es la siguiente ¿por qué el físico se debe remitir a los cuatro tipos de causas o explicaciones?

La respuesta a esta pregunta se encuentra en distintos pasajes de la misma Physica y en otras partes del corpus. El poder explicativo de la materia, por ejemplo, consiste en ser aquello a partir de lo cual se genera algo y que, a su vez, permanece al final del cambio. ${ }^{22}$ Ella, sin embargo, no explica lo que una substancia es actualmente, sino sólo lo que podría ser, i.e. lo que algo es en potencia. El principio que da cuenta del ser actual de las cosas es su forma. ${ }^{23}$ Sin embargo, estos dos principios no son capaces de explicar el desencadenamiento de un movimiento, pues para ello es necesaria la intervención de un agente. ${ }^{24}$ De esta manera, vemos cómo Aristóteles va ampliando el elenco de las causas: buscando los principios explicativos de un fenómeno o, como dice Carlo Natali, haciendo una deconstrucción de la noción platónica de causalidad. ${ }^{25}$ En lo que respecta a la causa final, no sería la excepción y buena parte de la propuesta

\footnotetext{
${ }^{20} \mathrm{Ph} .198 \mathrm{a} 22-24$.

${ }^{21}$ Cfr. Ph. 194a31-35.

${ }^{22}$ Cfr. Ph. 194b23-24.

${ }^{23}$ Cfr. Ph. 193a28-193b21.

${ }^{24}$ Cfr. Metaph. 984a16-27.

${ }^{25}$ Cfr. Natali 1999, 39-57.
} 
aristotélica aparece como respuesta a la pregunta ya mencionada de $P h$. II 8 , a saber, ¿por qué la naturaleza se encuentra entre las causas que son para algo?

Ahora bien, antes de pasar al detalle de la explicación de cómo se da la teleología en el ámbito de la naturaleza, quisiera hacer una precisión terminológica previa acerca del término phýsis y desde la cual quisiera formular una hipótesis. Para ello, retomo una distinción que hace Alejandro Vigo acerca de esta noción. ${ }^{26}$ En su opinión, el término "naturaleza" o phýsis puede aplicarse en dos niveles diferentes. El primero, sería un sentido distributivo-individualizador, que alude al nacimiento o la constitución o al modo de ser de algo particular. En segundo lugar, estaría el sentido colectivo-totalizador, que alude al conjunto de las cosas naturales o bien al orden que las comprende y regula. Éste último estaría más cerca del término kósmos referido a la totalidad del universo. Podría decirse que estos sentidos equivaldrían a la diferencia que se establece en el español al hablar de "la naturaleza de..." una determinada cosa, por un lado, y de "la naturaleza", a secas, por el otro. Vigo sostiene que, en el caso de Aristóteles, el sentido útilizado al referir una noción técnica de phýsis en las obras de filosofía teórica sería el primero, aunque algunas veces se apele al segundo en distintos pasajes del corpus. ${ }^{27} \mathrm{~A}$ mi manera de ver, esta distinción es importante para aclarar el alcance de la respuesta de II 8 y el sentido en el que se puede hablar de una dependencia entre la phýsis y la ousía eterna e inmóvil. Intentaré mostrar que mientras la argumentación del capítulo 8 del libro II de la Physica recoge el sentido distributivo-individualizador, el pasaje de Metaph. XII 7 se refiere, más bien, al sentido colectivo- totalizador. En aras de desarrollar esta idea, veamos a continuación la la respuesta ofrecida por Aristóteles en $P h$. II 8 y después nos detendremos, como ya se dijo, en la sobre la tesis de Metaph. XII 7.

\section{ARISTÓTELES Y LA DISCUSIÓN CON EL MATERIALISMO}

El tema de la causalidad final está presente en distintas obras del corpus aristotelicum, aunque el tratamiento temático más importante sobre la teleología natural se encuentra en el ya referido segundo libro de su Physica. Ahí encontramos, además, la definición aristotélica de la naturaleza, la teoría de las cuatro especies de causas y los cuatro modos causales, el tratamiento del azar y la fortuna, y termina justo con un posicionamiento acerca de la presencia de fines y de la necesidad en el mundo natural. Ahora bien, al inicio del capítulo 8 de este libro, Aristóteles señala que "debemos decir, primero, por qué la

\footnotetext{
${ }^{26}$ Cfr. Vigo 2013, 19-41.

${ }^{27}$ DC, 290a31; DA, 432b21; Metaph., 1005a33; y Pol., 1267b 28.
} 
naturaleza se encuentra entre las causas que son para algo". ${ }^{28}$ La respuesta a esta pregunta ocupará al filósofo griego en el resto del capítulo.

Simplicio destaca, en su comentario a II 8, el papel que juega esta pregunta para orientar el discurso de la parte final de $P h$. II, junto con la cuestión acerca de cómo se da la necesidad en la naturaleza. ${ }^{29}$ En una dirección similar, Filópono sostiene que una vez que se había dicho en la explicación del azar y la fortuna que la naturaleza actúa para algo, ahora tiene que probarlo. ${ }^{30}$ De ahí la pertinencia del tema. Tomás de Aquino, por su parte, al ordenar esos pasajes dice que después de haber explicado por qué el filósofo natural demuestra por todas las causas, expone aquí lo que supuso: que la naturaleza obra por un fin y que en cualquier ser (generado en sentido absoluto) lo necesario no deriva de las causas primeras en el ser: motor y materia, sino de las posteriores, la forma y el fin. ${ }^{31}$ Como podemos ver, las observaciones de Simplicio, Filópono y Aquino introducen distintos énfasis y matices, pero todos ellos son compatibles entre sí.

Aristóteles responde a la pregunta señalada tomando como punto de partida una discusión con cierta forma de materialismo y con otras posturas que presentan distintas objeciones a la presencia de fines en el mundo natural. Estos dos tipos de interlocutores nos permiten agrupar los argumentos en dos familias distintas que trataré de exponer a continuación. ${ }^{32}$

La primera parte de la argumentación de Aristóteles a favor de la inclusión de la naturaleza entre las causas finales consiste en una refutación de los argumentos que subyacen a una cierta forma de materialismo que toma como postura contraria a la suya. Los interlocutores en este apartado serían, principalmente, Empedócles y Anaxágoras. ${ }^{33}$

En aras de reconstruir la postura de sus interlocutores, Aristóteles pone en su boca la siguiente pregunta: "qué impide que la naturaleza no obre en vistas de un fin ni en vistas de lo mejor, sino del mismo modo en que Zeus hace llover no para que el grano crezca sino por necesidad". ${ }^{34}$ El planteamiento del tema en estos términos resulta útil para Aristóteles como punto de partida en la medida

${ }^{28}$ Ph.198b10-11.

${ }^{29}$ Cfr. Simplicio, In Ph., 369, 15.

${ }^{30}$ Cfr. Filópono, In Ph., 306, 15.

${ }^{31}$ Cfr. Santo Tomás de Aquino, In Ph. II, 1. XII, n. 171.

${ }^{32}$ En otro lugar he intentado mostrar la relevancia del carácter dialógico del discurso aristotélico en $P h$. II 8 tanto para abordar algunas dificultades de orden exegético, como otra más bien de tipo sistemático. Ahí se ensaya una primera reconstrucciçon de los argumentos aquí recogidos. Cfr. Ross 2006, 127-146.

${ }^{33}$ Cfr. Simplicio, In Ph., 369, 15.

${ }^{34}$ Ph. 198b16-19. 
que sugiere la posibilidad de dar razón de los fenómenos naturales prescindiendo de explicaciones teleológicas, i.e. la tesis opuesta a la que él quiere mostrar.

Un ejemplo del tipo de explicaciones que daría el partidario de esta versión del materialismo es que un fenómeno natural como la lluvia sucedería porque: "es necesario que lo que se evapora se enfríe y que lo enfriado descienda al convertirse en agua, pero que el grano crezca al producirse <este fenómeno> es algo accidental- , de modo semejante también si a alguien se le arruina la cosecha en el campo, no llueve para que la cosecha se pierda, sino que este hecho se produce por accidente." ${ }^{35} \mathrm{Si}$ vemos las causas que intervienen en la explicación contenida en este pasaje, veremos que se encuentran los elementos simples (en este caso, el agua y el aire) y su interacción con otros cuerpos (el sol). En sede aristotélica, sería una referencia solamente a causas materiales y eficientes. Por tanto, la conclusión que podría obtenerse de eso es que las cosas se producen por necesidad, pero no orientadas a un fin determinado, ya que todo ocurre como consecuencia simplemente de la naturaleza de los cuerpos simples y sus movimientos. Podríamos prescindir pues, de cualquier tipo de finalidad: el sol explica la evaporación del agua y el agua desciende una vez que se enfría. Esto sería una explicación suficiente de la lluvia y no sería necesario buscar un propósito, como mejorar o empeorar la cosecha, para completar la explicación. Eso, en todo caso, sería accidental o por azar.

Al explicar, aparentemente con éxito un fenómeno natural como la lluvia, el interlocutor citado por Aristóteles exigiría razones al defensor de la finalidad para no extender su explicación de ese fenómeno a toda la naturaleza: “¿qué impide que también sea así con las partes de los seres vivos en la naturaleza? Por ejemplo, es necesario que los dientes incisivos sean agudos y aptos para cortar. Las muelas, en cambio, deben ser anchas y planas para masticar el alimento. Por cierto, todo esto no se produce con este propósito sino por accidente; otro tanto ocurre en las demás partes en las que, al parecer, se da la finalidad "36 A partir del aparente éxito de la explicación de la lluvia al prescindir de fines, en este pasaje se da paso a la generalización de ese modelo explicativo para explicar otros fenómenos de la naturaleza. De acuerdo con este señalamiento y a pesar de la aparente orientación a fines de ciertos fenómenos, esto no sería el caso.

¿Cómo lidiar con la aparente orientación a fines que se da en la naturaleza? El interlocutor de Aristóteles diría que: "allí donde todas las cosas ocurren como si se hubiesen generado en vistas a un fin, entonces, esas cosas se conservan por estar espontáneamente bien constituidas. Y en cuanto a las cosas que no se dan de este modo, han perecido y continúan pereciendo como aquellos

\footnotetext{
${ }^{35} \mathrm{Ph} .198 \mathrm{~b} 19-23$.

${ }^{36}$ Ph. 198b23-29.
} 
bueyes de rostro humano de los que habla Empédocles." ${ }^{37}$ De esta forma, Aristóteles piensa que su interlocutor podría desacreditar la creencia del observador de la naturaleza que a primera vista presume una ordenación teleológica en ella. La referencia sería nuevamente Empédocles, quien sostendría una tesis cuyas resonancias para nuestro tiempo son muy sugerentes.

Dicho lo anterior la generalización de las tesis materialistas es posible sobre la base de suponer un principio de economía explicativa. Al respecto, Aristóteles señala que "Éste es el tipo de argumentación con la que se podrían plantear dificultades y, si hay alguna otra, es de este tipo". ${ }^{38}$ Como bien ha señalado David Charles, las dos tesis básicas del interlocutor materialista serían que (1) todo lo que ocurre por necesidad, ocurre como consecuencia de la naturaleza de los cuerpos simples y sus movimientos y que (2) cualquier cosa que no ocurra como resultado de la naturaleza y del movimiento de los cuerpos simples, ocurre por azar. ${ }^{39}$ Dado lo anterior, Aristóteles tiene ahora la carga de la prueba y tendría que decir si los principios explicativos alegados por los materialistas realmente lo son y, en caso de serlo, si dan razón suficiente de los fenómenos. Como veremos, la respuesta del filósofo griego no se formula para desacreditar el poder causal de la materia y la causalidad motriz, sino para mostrar que estos principios no dan una explicación completa de lo que sucede en la naturaleza.

\section{LA TELEOLOGÍA NATURAL EN PH. II 8}

Una vez planteados los términos de la discusión, revisaremos en el presente apartado los argumentos principales de Aristóteles a favor de la inclusión de la naturaleza entre las causas que son para algo. El primer texto que apunta en esa dirección es el siguiente:

"Es imposible, empero, que sea de esa manera, ya que éstas y todas las cosas naturales se generan de un mismo modo siempre o en la mayoría de los casos; pero no ocurre así en aquellos que es efecto del azar o de la espontaneidad. Porque no parecer ser efecto del azar o de la coincidencia fortuita el que llueva a menudo durante el invierno, pero sí que aquello ocurra en un día de perros. Tampoco parece parece un efecto del azar que haga calor en un día de perros pero sí en invierno. Consecuentemente, si tales cosas parecen generarse en virtud de una coincidencia fortuita o de un fin, y si no es posible que se generen por una coincidencia fortuita o por espontaneidad, deberán hacerlo, entonces, en virtud de un fin. Pero, ciertamente, todas las cosas descritas son por naturaleza, tal como

\footnotetext{
${ }^{37} \mathrm{Ph} .198 \mathrm{~b} 29-32$.

${ }^{38}$ Ph.198b33-34.

${ }^{39}$ Cfr. Charles 1995, 111.
} 
admitirían los sostenedores de la tesis antes expuesta. En consecuencia, la finalidad entra en las cosas que son y se generan por naturaleza". ${ }^{40}$

La reconstrucción propuesta de este argumento se puede hacer, según Simplicio, a partir de una disyunción y de un razonamiento que se podría reformular como un silogismo de la primera figura siguiendo las prescripciones de los Analíticos Primeros. ${ }^{41}$ La disyunción sería simplemente que lo que sucede por naturaleza se da por azar o con miras a un fin. A su vez, el resto del argumento se podría presentar de la siguiente forma:

(1) Nada que suceda frecuentemente se da por accidente.

(2) Algunas cosas que se dan por naturaleza se dan frecuentemente.

(3) Por tanto, algunas cosas que se dan por naturaleza no se dan por accidente.

(4) Por tanto, algunas cosas que se dan por naturaleza se dan en virtud de un fin.

Como puede verse, el argumento de Aristóteles está basado en la supuesta imposibilidad de que el azar sea causa de las regularidades observables en la naturaleza y, por tanto, hay que atribuirlas a un fin. Si bien la naturaleza del agua y su relación con el sol podrían dar una explicación, al menos parcial, de un fenómeno concreto, i.e. la lluvia, quedaría todavía pendiente la explicación de otro aspecto del fenómeno: su regularidad, i.e. por qué llueve frecuentemente en una temporada y por qué hace calor frecuentemente en otra. Si esto fuera algo que sucede rara vez, se podría explicar por el azar. Si esto sucediera siempre, se podría explicar por la naturaleza de los elementos. Sin embargo, esto sucede frecuentemente, de manera que debe buscarse un principio distinto.

El segundo argumento, introducido por Aristóteles ofrece una especie de definición de qué es la teleología en los siguientes términos: "Además, en las cosas que comportan un fin, hay algunas que se llevan a cabo primero y otras después, en vistas de dicho fin. En efecto, como se lleva a cabo una cosa, así también ella es por naturaleza; y en cuanto es por naturaleza, de ese modo se lleva a cabo, siempre y cuando no haya impedimento alguno. Pero ella se lleva a cabo en vistas de un fin y, consecuentemente, está por naturaleza ordenada a un 〈determinado〉 fin". ${ }^{42}$ La definición de teleología recogida en este pasaje se podría reconstruir de la siguiente forma, siguiendo la sugerencia de David

\footnotetext{
${ }^{40}$ Ph.198b34-199a8.

${ }^{41}$ Cfr. Simplicio, In Ph., 372, 25-373,12.

${ }^{42}$ Ph. 199a8-12.
} 
Charles: si $\mathrm{F}$ es el fin de la secuencia a1,...a3, entonces a1, a2 y a3 ocurren en vistas de F. ${ }^{43}$ Esta formulación es muy útil, en la medida que nos permite ver lo específico de las explicaciones teleológicas. Como explica Carlo Natali, la causa final nos permite distinguir algunas series causales dotadas de sentido, constantes y repetidas. La conexión de estas series sucede regularmente, y sus efectos últimos tienen un vínculo esencial con aquello que da comienzo a la cadena. ${ }^{44}$ Todo acontecimiento en el mundo tiene infinitas causas e infinitos efectos accidentales, pero sólo tiene una serie de causas y una serie de efectos en sentido propio. De manera que, un criterio exclusivamente empírico de sucesiones temporales de los acontecimientos no nos permitiría individualizar una serie de este tipo.

Ahora bien, ¿cómo saber si esta definición se cumple en el caso de la naturaleza? Aristóteles ofrece distintos ejemplos a favor de que esto es así. La primera de ellas es una analogía entre el arte y la naturaleza:

\begin{abstract}
"Por ejemplo, si una casa tuviese relación con los entes que se generan por naturaleza, se generaría del mismo modo en que ahora se genera por arte. Y pero si los entres naturales se generaran no sólo por la naturaleza, sino también por arte, se generarían del mismo modo que son por naturaleza. Una cosa, entonces, tiene por fin a la otra y, en suma, el arte lleva a cabo aquellas cosas que la naturaleza es incapaz de realizar y, además, imita a la naturaleza. Por tanto, si los entes artificiales son en vistas de un fin, es evidente que también lo serán los entes naturales. En efecto, en los entes artificiales y en los naturales lo posterior y lo anterior se encuentran entre sí en la misma relación" ${ }^{45}$
\end{abstract}

El argumento que encontramos este pasaje recoge la peculiar concepción que tenía Aristóteles de la relación entre arte y naturaleza. De acuerdo con el filósofo griego, si los entes artificiales están diseñados en función de un fin, es evidente que también lo serán los entes naturales. ¿Por qué? Porque el are imita a la naturaleza. Hay que entender el argumento, pues, a partir de la prioridad del orden natural que Aristóteles observa respecto de las obras de arte. Además, hay que considerar el valor explicativo que tienen las analogías para Aristóteles. En este caso, su uso no es sólo ilustrativo, sino también argumentativo. Como puede verse, es difícil entender por qué la constatación del modus operandi del arte sirve para inferir alguna característica del dinamismo natural, si no es a partir de esta relación de prioridad.

\footnotetext{
${ }^{43}$ Cfr. Charles 1995, 114.

${ }^{44}$ Cfr. Natali 1999, 51-52.

${ }^{45}$ Ph. 199a13-20.
} 
Ahora bien, si pasamos al segundo conjunto de ejemplos a favor de que la definición de teleología se cumple en el caso de la naturaleza, nos encontramos con que Aristóteles echa mano de algunas investigaciones de campo, es decir, de los resultados de sus observaciones de la naturaleza y cita algunos ejemplos verificables empíricamente.

"Y esto es particularmente manifiesto en aquellos otros vivientes que no actúan por arte, que no investigan ni deliberan. De aquí que algunos pongan en duda si las arañas, las hormigas $u$ otros animales semejantes obran en virtud de un intelecto o de alguna otra <capacidad $>$. Y el que procede así poco a poco comienza a creer que también en las plantas las cosas que son útiles se producen en vistas a un fin; v.gr. las hojas para proteger el fruto. Así pues, si la golondrina hace naturalmente su nido y en vistas de un fin, la araña su telaraña, las plantas producen sus hojas en vistas de los frutos, y si ellas afirman sus raíces debajo del alimento y no arriba, es evidente que una causa semejante debe haber en los entes que se generan y son por naturaleza”. ${ }^{46}$

El pasaje aquí citado nos presenta varios ejemplos a favor de que en la naturaleza lo anterior es por lo posterior, ya sea que hablemos del comportamiento o los cursos de acción que siguen los animales y las plantas. Dado que no obran por técnica, búsqueda o deliberación, Aristóteles concluye que lo anterior está ordenado a lo posterior por naturaleza. Como puede verse, en este caso lo anterior se refiere no sólo a la disposición de las partes de un ser natural respecto del todo, sino también a la orientación de la conducta en este tipo de substancias. ¿Qué diferencia habría entre estas observaciones y las que desacredita el interlocutor materialista? En principio, sería la definición de qué significa actuar en función de un fin y su carácter aspectual dentro de la explicación total de un fenómeno. La teleología sería parte de la deconstrucción de una noción de causalidad como la que se recoge en el Timeo de Platón.

Por último, una tercera instancia para mostrar que la definición anterior tiene lugar en el mundo natural consiste en retomar la distinción de los sentidos de "naturaleza" que aparecen en $P h$. II 1: "Y puesto que la naturaleza puede entenderse en dos sentidos, como materia y como forma, y dado que esta última es el fin y todo lo demás en vistas de un fin, la forma debe ser causa final”. ${ }^{47} \mathrm{El}$ argumento aquí recogido tendría una estructura muy sencilla:

\footnotetext{
${ }^{46}$ Ph. 199a20-30.

${ }^{47}$ Ph. 199a30-32.
} 
(1) La forma se comporta como fin.

(2) La naturaleza se puede entender como forma.

(3) Por lo tanto, la naturaleza se comporta como fin.

La justificación de la tesis (1) está, como ya adelantábamos, en $P h$. II $1 .{ }^{48}$ Ahí, Aristóteles identifica dos sentidos de naturaleza: como materia y como forma. $\mathrm{Al}$ primero se refiere diciendo que se trata "la primera materia sujeto de cada ser, que posee en sí misma el principio del movimiento y el cambio," ${ }^{49}$ es decir, lo que en principio constituye a cada cosa y que no se identifica con la forma, $v$.gr. el bronce con respecto de la estatua o la madera con respecto del lecho. En estos casos, tanto el bronce como la madera constituyen la estatua y el lecho. Apelando a Antifón, Aristóteles pone como signo de ello el hecho de que si se perfora el lecho, entonces brotarían yemas y retoños que no son lechos, ni sillas, ni mesas, sino madera. Esto se debe a que la forma artificial que hace que el lecho sea tal es accidental, como advertía el mismo Antifón. ${ }^{50}$

A la par de lo anterior, Aristóteles añade tres argumentos para justificar que la naturaleza también puede entenderse en un segundo sentido. Los mencionaré de manera sucinta. ${ }^{51} \mathrm{El}$ primero de ellos se puede reconstruir en los siguientes términos: si algo es conforme a la naturaleza y es natural en acto, entonces se llama naturaleza. Por otra parte, si decimos que algo es natural sólo potencialmente, entonces no se le llama naturaleza. Esto mismo sucede, por analogía, en el arte. Así pues, tenemos que si algo recibe la forma que integra su propia definición, entonces aquello será natural. De esta manera, dice Aristóteles, la naturaleza podría ser la especie y la forma de aquellos seres que tienen en sí mismos el principio del cambio y del movimiento. El segundo argumento, por su parte, arranca desde una premisa mayor implícita: todo lo que resulta por generación es naturaleza. La premisa menor, en cambio, puede tomarse de los dos ejemplos que cita el mismo Aristóteles. El primer ejemplo es que el hombre se hace a partir del hombre, mientras que el segundo es el ya citado ejemplo del leño que pudriéndose hace brotar yemas y retoños, produciéndose entonces más madera. Estos ejemplos pueden traducirse en términos más universales diciendo que la forma de los seres naturales resulta de la generación, como es el caso de la madera y del hombre. Si bien lo que se genera es el compuesto, un indicio de ello es la presencia de una forma distinta que le da identidad. Por este motivo, habría que decir que la forma es naturaleza. Por último, el tercer argumento sostiene como premisa mayor que la naturaleza

\footnotetext{
${ }^{48}$ Cfr. Ph. 193a30-193b21.

49 Ph.193a29-30

50 Cfr. Ph.193a12ss.

51 Ph.193a31-193b13.
} 
dicha como proceso de generación es un camino hacia la naturaleza y que la forma es el término del proceso de generación. Por tanto, la forma es naturaleza.

A partir de estos tres argumentos, Aristóteles concluye que la naturaleza no sólo es materia, sino también es forma. ¿Por qué? Entre otras razones, porque si alguien describe un ente a partir de la sola referencia a la materia, sólo describe lo que es en potencia. La forma, en cambio, explica la actualidad de la substancia. Al plantear la respuesta en estos términos, Aristóteles no parece estar restringiendo la respuesta al mundo biológico, pues la composición materia-forma es universal. Éste es uno de los pasajes en los que se puede advertir con claridad que Aristóteles lleva a cabo una cierta identificación de lo que él mismo denomina "forma" y "naturaleza" en el sentido distributivoindividualizador, como lo llama Alejandro Vigo. ${ }^{52}$

A partir de las instancias anteriores (la analogía con el arte, la estructura y los cursos de acción de los entes naturales y la doctrina de la composición hilemórfica de los entes), Aristóteles intentó mostrar que en la naturaleza, lo anterior es por lo posterior y, por tanto, se puede decir que está orientada conforme a fines. Si esto es así, no habría razones para negar que la naturaleza obra por un fin y lo alcanza, si nada se lo impide. De esta forma, cerraría la primera familia de argumentos a favor de la inclusión de explicaciones teleológicas en el estudio de la naturaleza.

Una vez expuesto lo anterior, Aristóteles abre una segunda línea de argumentación a favor de que la existencia de fines en el mundo natural es compatible con otras tesis que aparentemente la excluyen. Apelar a ellas para descartar la teleología sería, por tanto, improcedente. De entrada, Aristóteles pensaba que la existencia de errores en el mundo natural podría ser usada por alguien para negar la existencia de un orden teleológico en él. ${ }^{53}$ Sin embargo, esto no necesariamente es así, pues en otro contexto teleológico, como el arte, también hay errores y sabemos por experiencia que se obra para algo. Aristóteles pone el ejemplo del gramático y del médico. Éstos se equivocan y esto sucede actuando de cara a un fin. Incluso parecería que la posibilidad de identificar errores supone la estructura teleológica de las acciones. El gramático quiere escribir bien y el médico quiere curar, pero pueden equivocarse y no alcanzar el fin buscado. Esto mismo sucedería en la naturaleza. Los errores serían casos en los cuales no se alcanzó el estado al que apuntaba el proceso y resultó algo que no se buscaba. ${ }^{54}$

Una segunda postura con la que Aristóteles discute en este contexto es la de aquellos que niegan la teleología natural, porque la naturaleza no delibera. ${ }^{55}$

\footnotetext{
${ }^{52}$ Cfr. Vigo 2013, 19-41.

${ }^{53}$ Cf. Ph. 199a33-199b4

${ }^{54}$ Cfr. GA 767a 36-b15 y 770 b9-17.

${ }^{55}$ Cf. Ph. 199b26-33
} 
Sin embargo, Aristóteles piensa que la falta de deliberación en la phúsis no sería una razón suficiente para descartar la teleología natural. El ejemplo que da para ilustrar su posición es el ejercicio del arte, pues la deliberación no interviene en su ejecución y ello no le exenta de obrar con un fin determinado. ¿Cómo ejemplificar un caso de procesos teleológicos sin recurrir a acciones deliberadas pero tampoco a procesos naturales (lo cual sería una petición de principio)? Aristóteles encuentra en la ejecución del arte un caso intermedio. La fuerza de este argumento estaría en el hecho de que descansa en un tipo de actividad con la cual tenemos algún tipo de familiaridad y que no implica, según sus propias categorías, deliberación alguna. El artista ejecuta y no se detiene a deliberar cuál es el siguiente paso en su ejecución, como sería el caso, por ejemplo, de un buen pianista. El ejemplo de Aristóteles en el texto es que si el arte de construir barcos estuviese en la madera, haría lo mismo por naturaleza.

Una vez explicadas las dos familias de argumentos, Aristóteles deja por sentado que la naturaleza se cuenta entre las causas que son para algo y procede a estudiar el tema de la necesidad absoluta e hipotética en la Naturaleza. Ph. II 9 está consagrado a ese tema. He intentado mostrar en otro lugar que el modo de proceder en estos textos para probar que la naturaleza se cuenta entre las causas finales tiene, por lo menos, dos dimensiones: una es la disputa con el modelo de explicación materialista que hemos recogido aquí y otra son los ejemplos puntuales en los que se constata que se obra para algo. ${ }^{56}$ Esto puede tener su relevancia de cara a la discusión sobre los alcances o límites de las explicaciones teleológicas en la física aristotélica, pues una de las discusiones más recurrentes se centra en cuál es el papel que juega la explicación de la lluvia que aparece mencionado al inicio de II $8 .{ }^{57} \mathrm{Me}$ parece que si remitimos ese ejemplo a su trasfondo dialógico, tendríamos una explicación de por qué Aristóteles no se concentra en la refutación del citado ejemplo, sino en las coordenadas de pensamiento en las que se expone. Es decir, la lectura que sugerimos en este trabajo serviría para mostrar que la intención de II 8 no es dirimir si una entidad o un fenómeno concreto (v. gr. la lluvia), sucede para un fin determinado, sino cómo debemos explicar en general los procesos que ocurren en la naturaleza. La otra discusión, más bien, está reservada a otras obras. ${ }^{58}$

Ahora bien, como puede verse por los argumentos ya expuestos, a partir de $P h$. II 8 emerge un cuadro que arroja el primado de la teleología intrínseca en la concepción aristotélica en el ámbito de la filosofía natural. Esto, como bien señala Vigo, abre paso a otras discusiones, pues este tipo de teleología no basta

${ }^{56}$ Cfr. Ross 2016.

${ }^{57}$ Cfr. Ross 1936, 42-43; Charlton 1970, 120-126, Sorabji 1980, 147n85; Furley 1987, 177183; y Boeri 1993, 200-202 y Rossi 2010, 91-123.

${ }^{58}$ Cfr. Meteor. 346b20-36 y Boeri 1993, 200-202. 
para satisfacer los requerimientos que se exigen para dar cuenta de la constitución de un cierto "orden" de cosas, en especial, de un "orden cósmico". ${ }^{59}$ En este orden de ideas, Vigo añade que, por esta limitación, Aristóteles se vio obligado a introducir relaciones teleológicas de segundo y tercer orden en sede cosmológica. Ésta sería una diferencia importante con otras concepciones que, en su orientación inicial, tienen una inflexión holística más decidida y que ponen un énfasis mucho mayor en el papel de la teleología extrínseca, como podría ser la concepción estoica y la presentada por Platón en el Timeo.

\section{UNA DIGRESIÓN ACERCA DE METAPH. XII 7}

Una vez explicada la justificación de Aristóteles a favor de que debemos contar a la naturaleza entre las causas que son para algo y del primado del sentido distributivo-individualizador del término naturaleza en la filosofía de la naturaleza aristotélica, quisiera hacer una breve digresión acerca del pasaje de XII 7 . Es decir, acerca de la forma en la que el cielo y la naturaleza penden del principio descrito en dicho libro.

La tesis que quisiera defender a este respecto parte de la distinción de los sentidos de naturaleza mencionada anteriormente. Me parece que, así como Aristóteles recoge en $P h$. II 8 el sentido distribuitvo individualizador, en el pasaje de Metaph. XII 7 la referencia más bien es al sentido colectivo-totalizador. Para probarlo, debemos atender al contexto argumentativo de esas líneas.

¿A qué se refiere Aristóteles cuando dice que el cielo y la naturaleza penden de un principio como el ahí descrito? Recordemos que Aristóteles argumenta en Metaph. XII a favor de que es necesaria la existencia de una substancia eterna (porque de lo contrario todo sería corruptible, incluso el tiempo y el movimiento). ${ }^{60}$ Además, el principio del movimiento no puede ser un principio moviente y productivo que no actúa, ${ }^{61}$ su substancia es actividad, ${ }^{62}$ es inmaterial, ${ }^{63}$ es uno y mueve con un movimiento único sin ser movido ${ }^{64}$ y mueve como lo deseable y lo inteligible. ${ }^{65}$ De este principio es del que penden el cielo y la naturaleza. ${ }^{66}$ Líneas después dirá que la vida de este principio es como la mejor para nosotros por corto tiempo y que su actividad consiste en ser intelección de

\footnotetext{
${ }^{59}$ Cfr. Vigo 2013.

${ }^{60}$ Cfr. Metaph. 1071b3-11.

${ }^{61}$ Cfr. Metaph. 1071b12-17.

${ }^{62}$ Cfr. Metaph. 1071b17-20.

${ }^{63}$ Cfr. Metaph. 1071b20-22.

${ }^{64}$ Cfr. Metaph. 1072a25-26.

${ }^{65}$ Cfr. Metaph. 1072a26-27.

${ }^{66}$ Cfr. Metaph. 1072b13-14.
} 
intelección o pensamiento de pensamiento. ${ }^{67}$ Además, se le identificará con lo divino, con el bien y como algo perfecto, impasible, inalterable y separado. ${ }^{68}$

La reconstrucción tradicional de estas tesis, como es bien sabido, consiste en presentar a esta substancia eterna e inmóvil como causa final del movimiento eterno del cielo. La única actividad de dicho principio es ser intelección de intelección y la traslación circular del primer cielo sería una imitación de ella. A su vez, el cielo mueve y es movido, de manera que se comporta como causa de la sucesión eterna de generaciones y corrupciones que se da en el mundo sublunar. En otro lugar, he dicho por qué esta interpretación de Metaph. XII me parece correcta, a pesar de que en los últimos años ha recibido diferentes críticas. ${ }^{69}$

Ahora bien, en este orden de ideas, quisiera resaltar cómo la existencia de dicha substancia y de su descripción responde a la necesidad de explicar la eternidad del movimiento tanto en la Physica, como en la Metaphysica. En Physica VIII 5, encontramos la justificación de que todo lo que se mueve es movido por otro y que las series de motores y movidos no pueden proceder al infinito. Debe haber, por tanto, un Primer Motor. ${ }^{70}$ Sin embargo, la caracterización de dicho principio a partir de ese momento está ligada en el resto del libro a la explicación del movimiento eterno: (i) absolutamente inmóvil; ${ }^{71}$ (ii) impasible y sin mezcla; 72 (iii) uno, eterno y mueve con un movimiento único; ${ }^{73}$ y (iv) carece de magnitud. ${ }^{74} \mathrm{Al}$ mismo tiempo, si miramos los argumentos de XII 6-7, nos encontraremos con una situación similar. Si bien el tema principal de esos pasajes es la ousía y la definición de sus tipos, la descripción de los atributos de las substancia eterna e inmóvil está ligada también a la explicación de la eternidad del tiempo y el movimiento. De ahí que Aristóteles concluya que "tiene que haber algo que sin moverse mueva, que sea eterno, substancia y acto." 75 Dado este contexto explicativo, la lectura minimalista parece ser la más adecuada, pues no hay nada en el texto que nos haga pensar en una causalidad providente, dada la recurrencia del del explanans y del explanandum en las dos versiones de la teoría. El Motor Inmóvil o la substancia eterna e inmóvil, por una parte, y la eternidad del movimiento, por otra.

¿En qué sentido penden pues, el cielo y la naturaleza de ese principio? No en su acto de ser, no en su ordenación teleológica, pero sí en lo que respecta al movimiento eterno que se da en cada uno de ellos. En el caso del cielo, hablamos

\footnotetext{
${ }^{67}$ Cfr. Metaph. 1072b14-30 y 1074b29-35.

${ }^{68}$ Cfr. Metaph. 1072b4ss.

${ }^{69}$ Cfr. Ross 2016, 207-227.

70 Cfr. Ph.256a4-258b9.

${ }^{71}$ Cfr. Ph. 256b27-259a6.

72 Cfr. Ph. 258b10ss.

${ }^{73}$ Cfr. Ph. 256b20-27.

${ }^{74}$ Cfr. Ph. 266a12-b20.

${ }^{75}$ Metaph. 1072a25-26.
} 
de un movimiento circular y continuo. En el caso del mundo sublunar, hablamos de la sucesión eterna de generaciones y corrupciones, que no podría ser causada directamente por un motor absolutamente inmóvil, pero sí por la mediación de un motor movido. ${ }^{76}$ Ambos casos se refieren a entes naturales, es decir, a entes que tienen el principio de movimiento en sí mismos. De manera que al hablar genéricamente de la phýsis, podríamos pensar que el pasaje de XII 7 refiere más bien al sentido de "naturaleza" colectivo-totalizador y no el distributivo-individualizador. Por tanto, no sería legítimo apelar a dicho pasaje para construir un argumento que pusiera en conexión Metaph. XII y Ph. II 8 para argumentar que un dios o la substancia inmóvil pueda ser causa última de la ordenación a fines de la naturaleza en Aristóteles.

\section{CONCLUSIÓN}

He intentado mostrar una línea de reconstrucción minimalista de cómo se da la teleología en la naturaleza, según Aristóteles, así como de su valoración en el marco de algunas reconstrucciones intertextuales de su filosofía. Como vimos, Aristóteles presenta una argumentación a favor de la inclusión de explicaciones teleológicas en la naturaleza como respuesta al proyecto materialista de la tradición filosófica instaurada por los filósofos arcaicos. Ahí la estrategia consiste en mostrar fenómenos o algunos de sus aspectos, que no son reducibles a las propiedades de la materia y a los motores en su explicación. El modo de proceder, como pudimos comprobar, está dirigido a consignar cuáles son las causas que debe usar el físico en sus demostraciones, respondiendo al mismo tiempo la propuesta materialista que ofrece una alternativa más económica desde el punto de vista ontológico, aunque menos explicativa, según el Estagirita.

Ahora bien, si la reconstrucción que proponemos es correcta, podemos decir que el filósofo griego defiende en II 8 una noción distributiva-individualizadora de la naturaleza, que excluye la posibilidad de argumentar a favor de una conexión causal fuerte entre la substancia eterna e inmóvil de Lambda con la existencia de fines en la naturaleza. Si bien ésta es la substancia más perfecta, su rol explicativo consiste en mover siempre y de la misma forma a un motor que mueve y es movido, a saber, el cielo. Sea como causa final, como causa eficiente o como ambas, el explanandum en todo caso es el movimiento eterno. Así que mientras, la línea referida de XII 7 recoge el sentido colectivototalizador del término, la Physica explora más bien el sentido distributivoindividualizador de la phýsis, presentada por Aristóteles como un principio intrínseco de movimiento y reposo.

${ }^{76}$ Cfr. Ph. 260a.3-17. 


\section{BIBLIOGRAFIA}

Aphrodisias, Alexander of. 2003. On fate, trad. by Robert Sharples, Duckworth Publishers.

Aphrodisias, Alexander of. 1992. Quaestiones 1.1-2.15, trad. by Robert Sharples. Cornell University Press.

Aphrodisias, Alexander of. 1994. Quaestiones 2.16-3.15, trad. by Robert Sharples. Cornell University Press.

Aquino, Santo Tomás de. 1965. In octo libros Physicorum Aristotelis expositio, Edición y estudio de P. M. Maggiólo, Marietti, Turín-Roma.

Aristóteles: Física I-II. 1993. Introducción, traducción y comentario de Marcelo Boeri, Buenos Aires: Biblos.

Aristóteles. 1990. Metafísica. Trad. Valentín García Yebra. Madrid, Gredos.

Charles, D. 1995. "Teleological Causation in the Physics", en Judson, L. (ed.) (1995): pp. 101-128.

Charlton, W. 1970. Aristotle's Physics. Book I and II. Introducción, traducción y notas, Oxford: Clarendon Press.

Corso, L. - Soto, M. J. - Zorroza, I. (eds) 2013. Concepciones de la ley natural: medioevo latino y escolástica española e iberoamericana. Pamplona: EUNSA.

Furley, D. 1987. "The Rainfall Example in Physics ii 8", en Gotthelf-Lennox (1987): pp. 177-183.

Gotthelf, A. - Lennox, J. G. (eds.) 1987. Philosophical Issues in Aristotle's Biology, Cambridge.

Horn, Ch. (ed.) 2016. Aristotle’s Metaphysics Lambda - New Essays, De Gruyter, Boston/Berlin.

Judson, L. (ed.) 1995. Aristotle's Physics: A Collection of Essays, Oxford University Press.

MacIntyre, A. 1991. Historia de la Ética. Barcelona: Paidós.

Natali, C. 1999. "La noción de causa final en Aristóteles”. Anuario Filosófico 32, 39-57.

Ross, A. 2006. "La defensa del uso de explicaciones teleológicas en Física II 8". Tópicos 30 bis, 127-146.

Ross, A. 2013. "Metaphysics and Ethics in Aristotle: About Being and Duty", Rossi (ed.) (2013), pp.127-153.

Ross, A. 2016. "The causality of the Prime Mover in Metaphysics $\Lambda$ ", in Horn (2016), De Gruyter, Boston/Berlin, pp. 207-227.

Ross, W. D. 1924. Aristotle's Metaphysics. Oxford: Oxford University Press.

Ross, W. D. 1936. Aristotle's Physics. Oxford: Oxford University Press.

Rossi, G. 2010. “Aristóteles y la lluvia, una vez más”, Diánoia LV/65: 91-123.

Rossi, G. (ed.) 2013. Nature and the Best Life Exploring the Natural Bases of Practical Normativity in Ancient Philosophy, Hildesheim- Zúrich-Nueva York: Olms. 
Simplicius. 1882. In Aristotelis Physicorum Libros Quattor Priores Commentaria, ed. H. Diels, Berlin.

Sorabji, R. 1980. Neccesity, Cause and Blame. Perspectives on Aristotle's Theory, Ithaca, NY: Cornell University Press.

Vigo, A. 2013. "Naturaleza, finalidad y normatividad según Aristóteles. Apuntes para una reconstrucción sistemática", en Corso - Soto - Zorroza (eds); pp. 19-41. 